recibido: 04.01 .13 / aceptado: 05.03.13

\title{
Percepción de la publicidad en tabletas digitales. Estudio experimental comparado de las ediciones en iPad y en papel de un diario
}

Antonio Sanjuán Pérez

Universidade da Coruña

Natalia Quintas Froufe

Universidade da Coruña

Sandra Martínez Costa

Universidade da Coruña

\section{Palabras clave}

Tabletas digitales, iPad, publicidad, formatos, prensa, percepción del usuario

\section{Resumen}

Este artículo recoge los resultados de un estudio experimental sobre la percepción que tiene el usuario/lector de la publicidad en un diario impreso y en su versión adaptada a la tableta digital (iPad). El trabajo compara los niveles de recuerdo, visibilidad y rechazo de los anuncios en la tableta frente a los incluidos en soporte papel. El objetivo es el de identificar los formatos publicitarios más eficaces para la tableta digital, dadas sus especificidades de uso, interactividad y tamaño. El estudio se centra en la percepción de los formatos publicitarios para iPad con un menor nivel de interactividad, ya que muchos periódicos para tableta utilizan versiones enriquecidas del papel a los que incorporar links o fotografías y vídeos, en lugar de hacer una aplicación específica para estos dispositivos. Por lo tanto, los formatos estudiados son aquellos derivados de los habituales de la prensa en papel. Los resultados indican que por el momento en este tipo de formatos publicitarios no existen diferencias significativas en cuanto a la percepción que el usuario/lector tiene de ella. 


\title{
Advertising perception on iPad adapted press. A comparative study on iPad and newspaper editions
}

\section{Keywords}

Digital tablets, iPad, Advertising, Formats, Press, User's perception

\begin{abstract}
This article reports a study about users' perceptions of printed newspaper's advertising versus the adapted one to the digital tablet (iPad). This paper compares the recall, visibility and rejection levels of ads on the tablet and on printed press. Aim is to identify most effective advertising formats for the digital tablet, considering the specificities of use, interactivity and size. The study bases on the advertising Apps perception, especially those with a minor level of interactivity. Many newspapers for tablet use enriched versions of the paper and incorporate links or photos and videos instead of doing a specific application for these devices. Therefore, this study is about those derivatives of the habitual ones formats in web and newspapers. Results show that nowadays there's no significant difference in the way the user/reader perceives this kind of advertising formats.
\end{abstract}

\footnotetext{
Autores

Antonio Sanjuán Pérez [sanjuan@udc.es] es doctor en Ciencias de la Información por la Universidad Complutense. Profesor Titular de Comunicación Audiovisual y Publicidad desde 2003 en la Universidade da Coruña. Actualmente es vicedecano en la Facultad de Ciencias de la Comunicación, de la que fue decano entre 2003 y 2009.

Natalia Quintas Froufe [n.quintas.froufe@udc.es] es doctora en Publicidad y Relaciones Públicas por la Universidad de Vigo en 2008. Fue profesora ayudante en la Universidad de Alicante desde 2007 hasta 2010, cuando se incorporó como Ayudante Doctor en la Universidade da Coruña.

Sandra Martínez Costa [smartinez@udc.es] es doctora en Publicidad y Relaciones Públicas por la Universidade de Vigo desde 2008. Desde 2004 es profesora Ayudante Doctor de Comunicación Audiovisual y Publicidad en la Facultad de Ciencias de la Comunicación de la Universidade da Coruña.
} 


\section{La publicidad se adapta al iPad}

Las tabletas digitales, lideradas por el iPad de Apple, han irrumpido con fuerza en el mercado publicitario presentándose como un nuevo dispositivo con múltiples posibilidades para desarrollar estrategias publicitarias basadas en el permission marketing. La introducción y abaratamiento de costes de las tabletas digitales ha motivado la creciente adaptación de dichas estrategias a un nuevo soporte, distinto en concepto de diseño y uso de lo visto hasta ahora, pero similar en estrategia de marketing a otros dispositivos ya existentes en el mercado, como los smartphones.

Las tabletas de tipo iPad también han supuesto una revolución en el contexto de las aplicaciones informáticas y sobre todo en el sector editorial, que está tratando de adaptar los formatos de periódicos, revistas y libros a otros nuevos (Meier, 2011), interactivos, enriquecidos, multimedia... para conseguir así llegar a un público más joven y tecnológicamente más complejo. Este proceso de adaptación implica un cambio de concepto en la generación de contenidos, en la estructura de las redacciones de revistas y periódicos y, previsiblemente también, un cambio del modelo publicitario editorial tradicional (Flew, 2010). Este dato se confirma en el informe sobre el desarrollo de la sociedad de la información en España: «el éxito del iPad ha provocado una reacción en cadena alrededor de los medios de comunicación, entre los que se popularizan las versiones especiales adaptadas para la visualización en este dispositivo, consiguiendo en algunos casos gran rentabilidad, como es el caso de la revista Wired» (Informe eEspaña, 2011).

Las posibilidades de navegación, de compra en directo, de geolocalización y de respuesta en tiempo real de los compradores que ofrecen estos nuevos dispositivos, junto con la interactividad propia de los mismos, los convierten en soportes publicitarios de gran utilidad. A esto hay que añadirle el hecho de que sea el propio público el que elige la publicidad que quiere ver e incluso la que quiere tener instalada en su dispositivo móvil. Como recoge López, «el usuario no sólo quiere una mayor selección de la publicidad que recibe, sino que pretende recibir exclusivamente los anuncios que le interesan» (López, 2011). La personalización e hipersegmentación de la publicidad favorecen las estrategias publicitarias one to one, es decir, aquellas que son creadas de forma específica para el consumidor individual.

En esta fase inicial en la que la penetración del mercado de tabletas es aún reducida y, por lo tanto, con tipologías de usuarios todavía por formar es en la que deben averiguarse los modelos publicitarios más eficaces para el anunciante, para tratar de identificar y modificar aquellos que no lo son tanto. Entre otras cosas, porque la inversión en publicidad digital en Europa creció un 14,5\% en relación al año 2010 y las previsiones de crecimiento de la inversión publicitaria en estos medios son realmente alentadoras (Informe Adex, 2012), con lo que es altamente previsible una fuerte saturación publicitaria. 
Esta investigación forma parte de otra más amplia sobre el uso y desarrollo de aplicaciones publicitarias y de prensa para las tabletas digitales. En el marco de dicha investigación se ha procedido a una primera clasificación de los formatos publicitarios más comúnmente utilizados en tabletas digitales, entendiendo que existen dos grandes grupos diferenciados: los derivados de prensa y web; y los creados de forma específica y original como aplicaciones publicitarias por el propio anunciante (App publicitarias).

\section{Objetivos del estudio e hipótesis de partida}

El objetivo de este estudio es el de averiguar si la percepción y aceptación del consumidor hacia la publicidad insertada en la prensa en soporte papel difiere de la del iPad u otras tabletas digitales similares. El estudio aquí descrito se ha centrado en los contenidos publicitarios de la edición para iPad de La Voz de Galicia que se han comparado con la misma edición en papel. El diario, que no dispone de una versión similar para el sistema operativo Android, ocupa el décimo puesto en el ranking de los periódicos más leídos en España (EGM, 2012) y el quinto lugar en los periódicos de información general.

La adaptación de este periódico a la tableta consiste en una aplicación, o App, a modo de quiosco con el ejemplar del día en versión $p d f$ enriquecido con vídeos y fotografías. Además del ejemplar, el quiosco también cuenta con acceso a las trece ediciones ${ }^{1}$ del día, números atrasados hasta un mes, una carpeta con los ejemplares descargados, una galería de fotografías, otra de vídeos y una sección de última hora actualizada permanentemente siempre que el dispositivo esté conectado a Internet.

Se presenta una investigación experimental que plantea como objetivos generales los siguientes:

- Identificar los aspectos a mejorar en el diseño de la publicidad para el iPad.

- Identificar modelos publicitarios eficaces para los periódicos en el iPad.

- Valorar la experiencia del visionado o la navegación de la publicidad en el dispositivo en relación con la publicidad.

De forma específica para este estudio, los objetivos son:

- Medir el interés por el contenido publicitario en el papel frente al interés sobre el mismo contenido en el iPad.

- Medir el nivel de intrusismo que implica la publicidad en el papel con respecto de la que se hace en el iPad.

1 Las ediciones corresponden a La Coruña, Vigo, Ferrol, Santiago, Lugo, Ourense, Pontevedra, Arousa, Barbanza Carballo, Deza, A Mariña y Lemos. 
- Analizar de un modo comparativo el nivel de recuerdo, visibilidad y rechazo de la publicidad en relación al soporte en el que se inserta.

- Determinar si los formatos de publicidad en prensa deben adaptarse al iPad en cuanto a tamaño e interactividad.

Para alcanzar los objetivos para este estudio se parte de las siguientes hipótesis:

- H1. Una misma inserción publicitaria es más memorable en soporte iPad que en papel.

- H2. Una misma inserción publicitaria es más molesta en soporte iPad que en papel.

- H3. Una misma inserción publicitaria es menos visible en soporte iPad que en papel.

- H4. La visibilidad de las páginas impares es igual a la de las pares en el iPad.

- H5. La visibilidad de los formatos que ocupan la parte inferior de la página a cinco columnas es mínima en el iPad.

A la hora de plantear las hipótesis se han tenido en cuenta los siguientes condicionantes que afectan a la investigación:

- El formato del diario impreso es mayor que la tableta. La mayoría de los lectores colocan la tableta en sentido horizontal, ya que de hecho, la pantalla de inicio sólo se puede visualizar así condicionando de entrada el comportamiento del usuario. La consecuencia es que los lectores sólo visualizan de una vez media página del periódico. Por ello, es lógico esperar que los anuncios tipo faldón e incluso de media plana sean menos visibles, ya que implican el desplazamiento por la pantalla para llegar a la publicidad, sin el aliciente de seguir viendo contenido informativo. Algo semejante ocurre con los anuncios a toda plana: sólo se visualiza la mitad superior con el iPad en horizontal y se pasa de página sin ver la parte inferior del anuncio.

- Se ha considerado también un condicionante esencial en este estudio la novedad en el uso de la tableta, porque hace que el usuario se interese más por sus capacidades técnicas que por los contenidos del periódico, tanto informativos como publicitarios. Es lógico suponer que posteriormente el interés decrezca al habituarse al soporte.

Aceptando estos condicionantes, se inició el diseño de este estudio piloto que se relata a continuación.

\section{Método}

Para definir el método y como punto de partida de esta investigación se tienen en cuenta en primer lugar los estudios cuantitativos sobre el uso de las tabletas 
digitales (Business Insider, 2010; Eye-Square, 2010; IDC, 2011; In-stats, 2010; Change Wave Research, 2011); aunque son menos frecuentes también se acudió a los de carácter cualitativo (Bernal y Cabrera, 2011), en especial los referidos a los contenidos publicitarios (Eye-Square, 2010; Budiu y Nielsen, 2011; Howe, 2011).

También se toman como punto de referencia los resultados de estudios previos recientes sobre la eficacia de los anuncios en prensa escrita como los del U.S. Newspaper Advertising Bureau (U.S. Newspaper Advertising Bureau, 2012); también sobre la posición y el tamaño de la publicidad (Silk-Geiger, 1972) y sobre la influencia del uso del color (Starch, 1966; Sparkman-Austin, 1980); y otros más específicos sobre la publicidad en tabletas digitales (Howe, 2011).

Siguiendo el método de investigaciones previas (Budiu-Nielsen, 2011), para este estudio de carácter experimental se utilizó una muestra aleatoria de conveniencia de veintidós individuos de entre 18 y 24 años, estudiantes universitarios, de los que 13 eran mujeres y 9 hombres. La metodología seleccionada combinó técnicas de carácter cuantitativo (cuestionario) y cualitativo (focus group), tal como se explica a continuación. Se decidió que los participantes desconocieran el fin del estudio para evitar prejuicios en su comportamiento y opinión y se les preguntó tanto por el contenido publicitario como por la información que había estado leyendo. Cabe mencionar que para la mayoría de ellos fue la primera experiencia con este tipo de dispositivos.

Se determinó que once de los participantes leyeran el diario en el iPad y los once restantes en soporte papel. No se limitó el tiempo a ninguno de los dos grupos con el fin de no condicionar el ritmo de lectura de los participantes, que fueron separados en dos salas independientes.

Para el estudio se escogió la publicación para el iPad de La Voz de Galicia en su edición local de A Coruña y se hizo un primer análisis del tipo de publicidad insertada en el periódico, con el fin de extrapolar las tipologías publicitarias utilizadas. De ese primer estudio se simplificaron los formatos habituales a los siguientes:

- Doble página completa

- Doble media página

- Plana completa (5 columnas x 10 módulos)

- Robapáginas (entre 4 y 3 columnas x entre 6 y 8 módulos)

- Media página (5 columnas x 5 módulos)

- Faldones (5 columnas x entre 1 y 4 módulos)

- Modulares (otras combinaciones: 2x2, 2x3, 1x2, columna completa, doble columna, etc.) 
Los formatos identificados son los mismos en el iPad que en la versión en papel. El único formato diferente al del ejemplar impreso es el banner de patrocinio al iniciarse la aplicación. Aunque existe la posibilidad técnica de incorporar interacción y/o contenido enriquecido en los espacios publicitarios, no ocurrió en ninguno de los formatos analizados.

Al finalizar la lectura del periódico se les pasó un cuestionario en escala Likert combinado con preguntas sobre el nivel de recuerdo, visibilidad e intrusismo de los anuncios. Por otra parte, era de interés conocer si eran o no compradores de la marca, y si les había molestado (y, en su caso, cuánto) dicho anuncio. También se les preguntaba sobre el motivo por el que lo recordaba (el uso del color o fotografía, el tamaño, la ubicación en la página o el eslogan).

El cuestionario utilizado fue el mismo para los dos grupos de lectores, aunque, a los usuarios de iPad se les preguntaba además si habrían querido una mayor interactividad con el anuncio y de qué tipo (con botones interactivos, con enlaces web, con imágenes estáticas o con imágenes en movimiento).

Esta experiencia se llevó a cabo en dos días diferentes del mes de marzo de 2012. Inmediatamente después de cada sesión, se realizó un focus group con el fin de conocer las opiniones en profundidad de los participantes, así como otros aspectos no tratados en el cuestionario. Dichas sesiones fueron grabadas para su posterior visionado y transcripción con el fin de extraer conclusiones que complementaran los datos extraídos del cuestionario.

\section{Resultados}

Del estudio se extraen los siguientes resultados:

De las 51 marcas/anunciantes insertadas en $\mathrm{La}$ Voz de Galicia fueron recordadas quince por el conjunto de los participantes (véase tabla I). Cinco marcas (R, Gadis, El Corte Inglés, Darty y una guía de odontólogos) fueron recordadas más de una vez. Una marca (Gadis) fue recordada por cinco lectores de los dos soportes, tres en iPad y dos en papel (véase tabla II). 


\begin{tabular}{|c|c|c|c|c|}
\hline \multirow[b]{2}{*}{ Anunciante } & \multicolumn{2}{|c|}{$\begin{array}{l}\text { Marca recordada } \\
\text { según el soporte }\end{array}$} & \multicolumn{2}{|c|}{$\begin{array}{c}\text { Características del } \\
\text { anuncio }\end{array}$} \\
\hline & En el iPed & En papel & $\begin{array}{l}\text { Color o } \\
\mathrm{B} / \mathrm{N}\end{array}$ & $\begin{array}{l}\text { Tamaño en } \\
\text { módulos }\end{array}$ \\
\hline $\mathrm{R}$ & & $\mathrm{X}$ & Color & $4 \times 7$ \\
\hline Novagalicia Banco & & $\mathrm{X}$ & Color & $4 \times 7$ \\
\hline Supermercado Gadis & $\mathrm{X}$ & $\mathrm{X}$ & Color & $\begin{array}{l}\text { Página } \\
\text { completa }\end{array}$ \\
\hline Star center & & $\mathrm{X}$ & Color & $\begin{array}{l}\text { Página } \\
\text { completa }\end{array}$ \\
\hline Dolce Vita & & $\mathrm{X}$ & $\mathrm{B} / \mathrm{N}$ & $3 \times 3$ \\
\hline La Voz Autopromoción (Películas) & $\mathrm{X}$ & & Color & $5 \times 5$ \\
\hline $\begin{array}{l}\text { Universidad de Santiago de } \\
\text { Compostela }\end{array}$ & $\mathrm{X}$ & & $\mathrm{B} / \mathrm{N}$ & $5 \times 2$ \\
\hline El Corte inglés & & $\mathrm{X}$ & Color & $\begin{array}{l}5 \times 5 \text { Doble } \\
\text { página }\end{array}$ \\
\hline Vueling & $\mathrm{X}$ & & $\mathrm{B} / \mathrm{N}$ & $5 \times 3$ \\
\hline Epitouch Cirugia y Estética & & $\mathrm{X}$ & $\mathrm{B} / \mathrm{N}$ & $3 \times 2$ \\
\hline Darty & $\mathrm{X}$ & $\mathrm{X}$ & Color & $\begin{array}{l}\text { Página } \\
\text { completa }\end{array}$ \\
\hline $\begin{array}{l}\text { Guía de Odontólogos, Estomatólogos } \\
\text { y Cirujanos maxilofaciales }\end{array}$ & $\mathrm{X}$ & $\mathrm{X}$ & Color & $5 \times 4$ \\
\hline $\begin{array}{l}\text { Fundación Santiago Rey Fernindez- } \\
\text { Latorre }\end{array}$ & $\mathbf{x}$ & & Color & $5 \times 5$ \\
\hline Cruz Roja & $\mathrm{X}$ & & $\mathrm{B} / \mathrm{N}$ & $5 \times 2$ \\
\hline Aquagest & $x$ & & Color & $\begin{array}{l}\text { Página } \\
\text { completa }\end{array}$ \\
\hline
\end{tabular}

Tabla $\mathrm{n}^{0} 1$ : Marcas recordadas según soporte y formato utilizado.

Los formatos más recordados fueron los de robapáginas, página completa y faldón a doble página, todos ellos en color (véase tabla 1). Cabe destacar que para llegar al faldón a doble página en la tableta es necesario el desplazamiento del usuario por la pantalla. Además, la doble página pierde su sentido en la tableta, ya que no existe la paginación par-impar, y por lo tanto se rompería el orden de lectura de la publicidad en este formato. Este hecho afectó en el caso de uno de los anuncios (El Corte Inglés), recordado por los lectores de papel, pero que pasó totalmente desapercibido en los del iPad.

\begin{tabular}{|c|c|c|c|c|c|}
\hline $\begin{array}{c}\text { Recordaron } \\
\text { la marca }\end{array}$ & $\begin{array}{c}\text { Gadis } \\
\text { (página } \\
\text { completa a a } \\
\text { color) }\end{array}$ & $\begin{array}{c}\text { R } \\
\text { (robapáginas } \\
\text { color) }\end{array}$ & $\begin{array}{c}\text { El Corte } \\
\text { Inglés } \\
\text { (media } \\
\text { doble } \\
\text { página) }\end{array}$ & $\begin{array}{c}\text { Guía de } \\
\text { odontólogos de } \\
\text { A Coruña } \\
(5 \times 4)\end{array}$ & $\begin{array}{c}\text { Darty } \\
\text { (página } \\
\text { completa a } \\
\text { color) }\end{array}$ \\
\hline $\begin{array}{c}\text { Lectores de } \\
\text { papel }\end{array}$ & 2 & 2 & 3 & 1 & 1 \\
\hline $\begin{array}{c}\text { Letores de } \\
\text { iPad }\end{array}$ & 3 & - & - & 1 & 1 \\
\hline
\end{tabular}

Tabla $\mathrm{n}^{\circ} 2$ : Marcas recordadas por más de un lector y soporte utilizado.

En cuanto a las variables que afectaron al recuerdo de las marcas, la posición y el uso del color en el papel fue más determinante para captar la atención que en la tableta, mientras que otras características como el tamaño y la fotografía están prácticamente equiparadas en ambos soportes. El eslogan fue mencionado como elemento motivador predominante en el papel, mientras que en el iPad pasó desapercibido. 


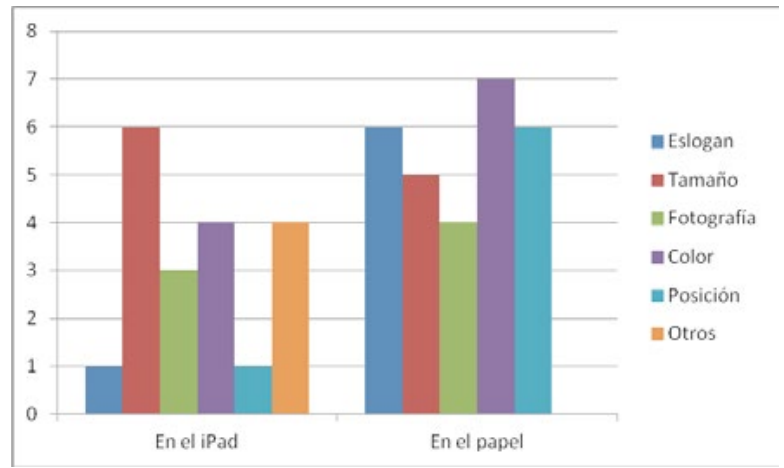

Figura $n^{0} 1$ : Elementos que incitaron el recuerdo de las marcas, según soporte.

En relación a las preguntas específicas para los lectores en iPad, de todos los participantes del estudio, tres afirmaron que no querían una mayor interactividad en la publicidad que habían visto. En cambio, cinco participantes que utilizaron el iPad demandaron inserción de botones y enlaces web que les permitiesen interactuar con el anuncio. De ellos, un amplio porcentaje no recordaba ninguna de las marcas que había visto leyendo el periódico, pero a pesar de ello, consideraba que era un valor imprescindible interactuar con los contenidos publicitarios del periódico.

En definitiva, y en relación a las hipótesis planteadas en el inicio del artículo, el único aspecto que ha influido en el recuerdo de los anuncios es el formato, no el soporte (H1). En el caso de los anuncios a página completa las marcas fueron recordadas por igual en el papel y en el iPad. Sin embargo, destaca el caso de los formatos de faldón, más llamativos en el periódico en papel, pero que pasaron inadvertidos en el iPad, lo que confirma las hipótesis H3 y H5 del estudio. En lo relativo a la intrusión que ha supuesto para el lector la inserción publicitaria en los distintos soportes (H2), debe tenerse en cuenta que los formatos que resultaron más llamativos en el papel pasaron casi inadvertidos en el iPad, pero en general los lectores no estimaron la intromisión de forma negativa. También cabe resaltar que las páginas impares no tuvieron una mayor visibilidad (H4) en el iPad que en el papel y la doble paginación de la publicidad carece de sentido, ya que se rompe la coherencia visual y textual del anuncio.

Durante la realización de los focus group los participantes, inducidos por el moderador, mostraron que el nivel de recuerdo de los anuncios en el papel era mayor de lo evidenciado en los cuestionarios. Se detectó también que algunos usuarios de iPad abandonaron la lectura del periódico en pdf y navegaron por secciones de la aplicación del periódico ausentes de publicidad. Por otra parte, también quedó en evidencia la decepción de los usuarios ante la publicidad insertada, la cual consideraban que debía gozar de un mayor grado de interactividad. 


\section{Conclusiones}

Por lo tanto, y a la vista de los resultados, se pueden extrapolar como conclusiones generales que:

Los participantes que leyeron el periódico en el iPad han demandado la inserción de interactividad en la publicidad. Sin embargo, debe tenerse en cuenta que no eran usuarios habituales de iPad y por lo tanto, sería necesario confirmar esta demanda en usuarios con experiencia. El nuevo usuario está más predispuesto a hacer clic en todo lo que aparece en la publicación. En esta fase de lanzamiento, incluir más interactividad en la publicidad es una oportunidad para atraer la atención y habituar al consumidor a las nuevas posibilidades de interacción tales como la compra en directo del producto anunciado. No obstante, la incorporación de interactividad a estos anuncios puede ser prescindible, al menos a la espera de una mayor adaptación del usuario al soporte, pero una adecuación del formato al tamaño de pantalla y la mejora en la definición y la calidad de las imágenes puede resultar más urgente.

Por otra parte, los formatos tradicionales de publicidad en prensa no resultan en general más molestos al lector cuando son adaptados al soporte iPad. A pesar de ello tampoco son especialmente llamativos, incluso cuando se ha utilizado un formato a pantalla completa y a todo color. Con frecuencia el usuario trataba de interactuar con el anuncio, pero a pesar de ello no se fijaba en la marca. Las veces que prestó atención a la marca no notó que ésta le molestase especialmente.

Y por último, los formatos publicitarios que ocupan la parte inferior de la plana deben evitarse si se busca la visibilidad en el iPad, especialmente si los lectores la utilizan en horizontal. Es recomendable utilizar más anuncios verticales y que vayan acompañados de información. De la misma manera que en los periódicos de formato sábana la maquetación de la página tenía en cuenta el número de dobleces que hacía el lector, es necesario tener en cuenta el formato de los anuncios en función de la pantalla de la tableta y de su posición en vertical y en horizontal. Como ya se advirtió, muchos de los formatos publicitarios en prensa escrita ni siquiera llegan a ser vistos por el usuario cuando son adaptados al iPad. Por ejemplo, en el caso de los faldones, es frecuente que el lector del periódico no continúe navegando por la pantalla hacia la parte inferior de la página cuando ya ha acabado la información, lo que hace que el anuncio sea prácticamente invisible.

En el caso concreto del medio seleccionado, La Voz de Galicia se sigue imprimiendo con la mayoría de las páginas en blanco y negro por cuestiones de coste y esa publicidad tiene tarifas diferentes a las de la publicidad en color. En el iPad es posible elaborar todas las páginas, incluida la publicidad, a todo color sin incremento de coste. Sin embargo, el diario no aprovecha esta oportunidad de lograr un mayor impacto visual tanto en sus informaciones como en su publicidad e incrementar, llegado el caso, sus tarifas. 
En general, las diferencias de percepción de la publicidad en iPad y en papel no son altamente significativas en el tipo de anuncios analizados para esta investigación, por lo que si el anunciante quiere que su publicidad destaque más en la tableta digital que en el papel deberá adaptar el formato al soporte. Por ello, si se pretende una mayor notoriedad y visibilidad para la publicidad en la prensa adaptada al iPad, es necesaria la adecuación y creación de nuevos formatos publicitarios específicos y no simplemente el traslado de los tradicionales de la prensa escrita, ya que estos carecen de efectividad por las particularidades del soporte en el que se insertan.

La limitación de este estudio piloto de carácter experimental, ligada al número de participantes y al medio seleccionado, impide extrapolar cualquiera de los datos presentados pero sí permite tener una visión, todavía incipiente, de la percepción de la publicidad por parte de los lectores y usuarios.

\section{Bibliografía}

Abuín Vences, Natalia (2009). La eficacia publicitaria en prensa digital. Madrid: Fragua.

Adelantado, Eulalia y Martí, José (2012). Nuevos formatos publicitarios y telefonía móvil: los mobile advergames. En: Zer, 33, 31-45.

AIMC. «Estudio General de Medios. Resumen General de Resultados EGM» (abril de 2011 a marzo de 2012). Disponible en: http://www.aimc.es/ (12/04/2012).

Bernal, Ana Isabel y Cabrera, María Ángeles (2011). Tabletas, smartphones, medios online, contenidos y consumos. En: I Congreso Internacional Comunicación y Educación: Estrategias de Alfabetización Mediática.

Budiu, Raluca \& Nielsen, Jacobson (2011). Usability of iPad Apps and Websites. Disponible en: http://www.nngroup.com/reports/mobile/iPad/ (07/07/11).

Business Insider. (2010). «iPad Survey Results: Everything you need to know about how people use the iPad». Disponible en: http://www.businessinsider. $\mathrm{com} /(07 / 07 / 12)$.

Change Wave Research (2011). «iPad Demand Takes Tablet Industry by Storm And New Owners Weigh in on Latest Apple Tablet». Disponible en: http://www. changewaveresearch.com/research-reports (05/03/12).

IDC (2011). Nearly 18 Million Media Tablets Shipped in 2010 with Apple Capturing 83\% Share; eReader Shipments Quadrupled to More Than 12 Million. Disponible en: http://www.idc.com/about/viewpressrelease.jsp? containerId=prU S22737611 \&sectionId=null\&elementId=null\&pageType=SYNOPSIS $(20 / 03 / 12)$. 
Eye Square (2012). «iPad -the new way of reading: Goodbye Gutenberg». Disponible en: http://www.eye-square.com/fileadmin/docs/presentations/press/ eye-square-iPad-Studie-2010-praesentation-en.pdf (05/01/12).

Gimeno, Manuel (2011). Informe eEspaña: informe anual sobre el desarrollo de la sociedad de la información en España. Madrid: Fundación Orange.

IAB Europe (2012). Informe ADEX Benchmark 2011. Disponible en: http:// www.iabspain.net/wpcontent/uploads/downloads/2012/07/iabeurope_adex_ benchmark_2011.pdf (07/07/12).

Howe, Patrick (2011). Of ads and apps: the influence of advertising on user attitudes toward tablet newspapers. Tesis, Universidad de Missouri-Columbia. Disponible en: https://mospace.umsystem.edu/xmlui/bitstream/handle/10355/11176/research.pdf? sequence=3 (07/09/12).

Hughes, Jeffrey (2012). Marketing de aplicaciones iPhone \& iPad. Madrid: Anaya multimedia.

In-stats (2011). Tablet Shipments to Surpass 58 Million Units in 2014. Disponible en:http://www.instat.com/press.asp?ID=3053\& sku=IN1104919ID (07/03/12].

Flew, Terry (2010). What will the Apple iPad deliver for newspapers?. En: Record of the Communications Policy and Research Forum 2010. Diponible en: http://eprints.qut.edu.au/' (01/09/2012).

López, David (2011). El digital out of home (dooh) o digital signage, el otro marketing digital. En: Trípodos, 28, 25-34.

Meier, Christian (2011). Erlösmodelle im E-Publishing: Wie sich Medien auf Tablets und Smartphones neu erfinden Könnnen. Hamburg: Kress.

Sanjuán, Antonio, Nozal, Teresa y González, Ana (2012). Prensa para iPad: Usabilidad, interactividad y multimedia en El País y Reforma. En: Revista Mexicana de Comunicación, 131. Disponible en: http://mexicanadecomunicacion.com. $\mathrm{mx} / \mathrm{rmc} / 2012 / 09 / 03 /$ prensa-para-iPad-usabilidad-interactividad-y-multimediaen-el-pais-y-reforma/ (01/09/2012).

Silk, Alvin y Geiger, John F (1972). Advertisment size and the relationship between product usage and advertising exposure. En: Journal of Marketing Research, IX (1), 22-26.

Sparkman, Richard y Austin, Larry (1980). The Effect on Sales of Color in Newspaper Advertisements. En: Journal of Advertising, 9, 39-42.

Starch, Daniel (1966). Measuring advertising readership and results. McGrawHill: Nueva York.

Tognazzi, Alberto (2012). Las transformaciones de los contenidos audiovisuales y la influencia de los dispositivos móviles en el nuevo escenario transmedia. En: Anàlisi, 81-95. 
U.S. Newspaper Advertising Bureau. Newspaper Multiplatform Usage. Disponible en: http://www.naa.org/Topics-and-Tools/Miscellaneous/Research/2012/Newspaper-Multiplatform-Usage.aspx (01/09/2012).

\section{Referencia de este artículo}

Sanjuan Pérez, Antonio, Quintas Froufé, Natalia y Martínez Costa, Sandra (2013). Percepción de la publicidad en tabletas digitales. Estudio comparado de las ediciones en IPad y en papel de un diario. En: adComunica. Revista Científica de Estrategias, Tendencias e Innovación en Comunicación, $\mathrm{n}^{\circ} 5$. Castellón: Asociación para el Desarrollo de la Comunicación adComunica, Universidad Complutense de Madrid y Universitat Jaume I, 197-209. DOI: http://dx.doi. org/10.6035/2174-0992.2013.5.12 